\title{
Roles of immune inhibitory molecule B7-H4 in cervical cancer
}

\author{
SAI HAN, YI LI, JINGJING ZHANG, LU LIU, QIAN CHEN, QIUHONG QIAN, \\ SHAN LI and YOUZHONG ZHANG
}

Department of Obstetrics and Gynecology, Qilu Hospital of Shandong University, Jinan, Shandong 250012, P.R. China

Received October 17, 2016; Accepted December 2, 2016

DOI: 10.3892/or.2017.5481

\begin{abstract}
Recent studies have reported that the immuneregulatory protein B7-H4 is highly expressed in various types of cancer, but little is known concerning its roles in cervical cancer. In the present study, we investigated the expression of B7-H4 in human tissues and serum samples, and explored the effects of B7-H4 on proliferation, apoptosis, migration and invasion of cervical cancer cell lines, including SiHa and HeLa. We found that B7-H4 was mainly located in the cytoplasm of cervical cancer cells as determined by immunofluorescence staining. Serum B7-H4 (sB7-H4) was overexpressed in patients with cervical intraepithelial neoplasia (CIN) and cervical cancer, and the area under the ROC curve (AUC) was 0.955. There was no statistical significance between B7-H4 expression and clinicopathological factors in cervical cancer tissue samples. B7-H4 promoted the proliferation of $\mathrm{SiHa}$ and $\mathrm{HeLa}$ cells, and protected them from apoptosis, which was related to the upregulation of E7, phosphorylated Rb (pRb), E2F, P16, P21, Bcl-2 and the downregulation of Rb, cleaved PARP and cleaved caspase- 3 as determined by western blotting. In addition, B7-H4 increased the ability of cell migration and invasion by targeting angiogenic factors, matrix metalloproteinase (MMP)-2, MMP-9 and vascular endothelial growth factor (VEGF) as determined by RT-PCR. Our findings revealed that $\mathrm{B} 7-\mathrm{H} 4$ has the potential to be a useful prognostic marker. In addition, B7-H4 plays important roles in proliferation, apoptosis, migration and invasion, indicating that $\mathrm{B} 7-\mathrm{H} 4$ can serve as a new therapeutic target for cervical cancer.
\end{abstract}

\section{Introduction}

Cervical cancer is the second most common cancer in women following breast cancer in developing countries (1). In China, the incidence of cervical cancer is 28.2/1,000 among the 30-44 female age group according to recent statistical data (2). It has now been established that persistent infection of

Correspondence to: Dr Youzhong Zhang, Department of Obstetrics and Gynecology, Qilu Hospital of Shandong University, 107 Wenhua Xi Road, Jinan, Shandong 250012, P.R. China

E-mail: zhangyouzhong@sdu.edu.cn

Key words: B7-H4, cervical cancer, diagnosis, proliferation, apoptosis, migration, invasion high-risk human papillomavirus (HPV) is the major risk factor of cervical cancer, and the expression of HPV oncogenes E6 and E7 play an important role during neoplastic growth (3). However, a majority of sexually active women get transient infections more than once in their lifetimes, which causes the low specificity of HPV testing (4). The delayed use of the HPV vaccine in developing countries incurred a large amount of cervical cancer patients. Consequently, there is an urgent need for specific diagnostic methods and therapeutic approaches against cervical cancer.

The B7 protein superfamily provides positive and negative signals that modulate immunological functions, including B7-1 (CD80), B7-2 (CD86), PD-L1 and PD-L2, which bind to coinhibitory molecules CTLA-4 and PD-1, downregulating T-cell function (5). B7-H4 was discovered as a B7 family member in 2003. It is also known as B7x, B7S1, VTCN1 and DD-O110 (6), and was reported to serve as a negative modulator in antitumor responses by inhibiting the functions of $\mathrm{CD}^{+}$and $\mathrm{CD}^{+}$ cells (7-9). However, there have also been studies reporting the positive regulation of the antitumor immunity of B7-H4, and it is presumed that there are at least two independent receptors which are differentially expressed under certain conditions causing the inverse effects of B7-H4 $(10,11)$.

B7-H4 is widely expressed in many types of cancer, including lung and breast cancer, renal cell and colorectal carcinoma, and ovarian cancer (12-16). This molecule has demonstrated great diagnostic potential in ovarian cancer particularly $(17,18)$. To evaluate the prognostic role of B7-H4 in cervical cancer, we sampled the tumor serum and tissue samples obtained from cervical intraepithelial neoplasia (CIN) and cervical cancer patients and examined their B7-H4 expression levels. Through gene silencing or overexpression of B7-H4 in cervical cancer cell lines, we described the effects of B7-H4 on proliferation, cell cycle arrest, apoptosis, migration and invasion of cancer cells.

\section{Materials and methods}

Serum and tissue samples of patients. Thirty-four normal matched controls (healthy or uterus benign tumor cases), $20 \mathrm{CIN}$ and 100 cervical cancer patients (providing serum or tissue samples) admitted to Qilu Hospital of Shandong University were enrolled in the present study, between 2015 and 2016. The CIN and cervical cancer patients were staged according to the 2009 FIGO staging guidelines (19). For all these patients, the records containing the age, HPV infection 
history, TCT, colposcopy and pathology were examined. The present study, was approved by the Ethics Committee of Qilu Hospital.

Cell lines and culture conditions. The human cervical carcinoma (HeLa, SiHa and CaSki) and E6/E7 immortalized human cervical epithelial (H8) cell lines were obtained from the Cancer Center Laboratory of Shandong University (Jinan, Shandong, China). The HeLa and H8 cells were maintained in Dulbecco's modified Eagle's medium (DMEM), SiHa cells were maintained in minimum essential medium (MEM), CaSki cells were maintained in Roswell Park Memorial Institute (RPMI)-1640 medium, and all of the media (Hyclone Laboratories, Logan, UT, USA) were supplemented with $10 \%$ fetal bovine serum (FBS; Gibco, Sydney, Australia). The four cell lines were incubated in a humidified atmosphere at $37^{\circ} \mathrm{C}$ with $5 \% \mathrm{CO}_{2}$. In addition, we collected cervical fall off epithelia tissue from healthy women volunteers as normal uterine cervix (NUC) cells.

Immunofluorescence staining. The SiHa and HeLa cells were washed there times with phosphate-buffered saline (PBS) and fixed with $4 \%$ paraformaldehyde for $15 \mathrm{~min}$. After permeation with $0.2 \%$ Triton $\mathrm{X}-100$ in PBS for $10 \mathrm{~min}$, the cells were immunostained with rabbit anti-B7-H4 monoclonal antibody (1:100 dilution in PBS; Abcam, Cambridge, MA, USA) at $4^{\circ} \mathrm{C}$ overnight. The cells were washed three times with PBS for $10 \mathrm{~min}$ each, and incubated for $1 \mathrm{~h}$ with a secondary goat anti-rabbit IgG antibody (1:200 dilution in PBS; Zhongshan Jinqiao Biotechnology Co., Ltd., Beijing, China) at room temperature. Cells were washed in 4',6-diamidine-2'-phenylindole dihydrochloride (DAPI) (Yusen Biotech Inc., Shanghai, China) for 3 min away from the light. Microscopic observations were performed using the Olympus IX51 inverted microscope.

Western blotting. The transfected cells were washed three times with PBS and lysed on ice using radio immunoprecipitation assay buffer (RIPA; Beyotime Institute of Biotechnology, Haimen, China) with $1 \%$ phenylmethylsulfonyl fluoride (PMSF) and $1 \% \mathrm{NaF}$ for $30 \mathrm{~min}$. The normal and tumor tissues were cut into pieces using tissue scissors, and homogenized by a tissue grinder (Tiangen, Beijing, China). The reagents were added into the tissue homogenates as aforementioned. Cell and tissue lysis were centrifuged at $12,000 \mathrm{rpm}$ for $10 \mathrm{~min}$ at $4^{\circ} \mathrm{C}$ and the protein extracts $(30-50 \mu \mathrm{g})$ were loaded in each lane and separated by sodium dodecyl sulfate-polyacrylamide gel electrophoresis, and transferred to polyvinylidene fluoride membranes (Millipore, Darmstadt, Germany). The membranes were blocked with 5\% skim milk and probed with anti-E2F (ProteinTech Group, Inc., Chicago, IL, USA), anti-B7-H4, anti-phosphorylated Rb-(pRb), anti-P16, antiP21, anti-Bcl-2, anti-cleaved PARP, anti-cleaved caspase-3 (Abcam) and anti-GAPDH [Cell Signaling Technology (CST), Danvers, MA, USA] antibodies. The membranes were then incubated with anti-rabbit and anti-mouse IgG (Millipore), and detected by enhanced chemiluminescence (ECL) using ImageQuant LAS 4000 (GE Healthcare Life Sciences, Logan, UT, USA). The results were analyzed by ImageJ software (NIH, Bethesda, MD, USA).
Reverse transcription and real-time PCR. The cellular RNA from the tumor cells and tissue samples was extracted using TRIzol reagent (Life Technologies, Carlsbad, CA, USA) and the cDNA was synthesized from 3-5 $\mu \mathrm{g}$ of RNA using M-MLV reverse transcriptase (Invitrogen, Shanghai, China) according to the manufacturer's instructions. In addition, primer-probe sets for qPCR for each gene were designed using the PrimerBank and are listed in Table I. The data were collected using the StepOnePlus ${ }^{\mathrm{TM}}$ software (Applied Biosystems, Shanghai, China) and quantified using the $2^{-\Delta \Delta C t}$ method.

ELISA assay. The serum samples for the detection of B7-H4 were kept frozen at $-80^{\circ} \mathrm{C}$ until use. The ELISA kit for the detection of B7-H4 was obtained from LifeSpan Biosciences, Inc. (Seattle, WA, USA) and used according to the manufacturer's recommendations.

Immunohistochemical staining and evaluation. The tissues were cut into 4- $\mu \mathrm{m}$ sections. Standard SP immunohistochemistry (Maxim, Fuzhou, China) was performed with the B7-H4 antibody (1:500 dilution in PBS; Abcam) and a Polink-2 Plus Polymer HRP Detection System (ZSGB-BIO, Beijing, China). The detection of B7-H4 in cancer cells was assessed by two independent investigators. Quantifications were recorded as follows: $<10 \%$ positive cells, $0 ; 10-25 \%, 1 ; 26-50 \%, 2$; $51-75 \%, 3 ;>75 \%$ positive cells, 4 . Staining intensity was scored as: absent, 0 ; weak, 1 ; moderate, 2 ; and strong, 3 . The final score was the multiplication of the quantification and staining intensity. A final score of $0-1$ was classified as negative, and $\geq 2$ was considered positive.

Silencing of the B7-H4 gene in SiHa cells. The small interfering RNA sequences targeting human B7-H4 (Si-B7-H4) and its control sequence (Si-control) were designed by GenePharma Co., Ltd. (Shanghai, China). The SiHa cells were seeded in a 6 -well plate with $4 \times 10^{4}$ cells $/ \mathrm{ml} /$ well. Twenty-four hours later, cells were transfected with $50 \mathrm{nM}$ of the Si-B7-H4 or control sequences using Lipofectamine 2000 (Invitrogen Life Technologies). The transfected cells were harvested $48 \mathrm{~h}$ post-transfection for the follow-up experiments. The Si-B7-H4 and $\mathrm{Si}$-control sequences are listed in Table I.

Overexpression of the B7-H4 gene in HeLa cells. The B7-H4 plasmid was designed by GenePharma Co., Ltd., and the lentiviral vector pLenti-C-Myc-DDk-IRES-Puro (7.6 kb) under the control of a cytomegalovirus promoter was obtained from Vector Gene Technology Co., Ltd. (Beijing, China). After the transfection and virus package, puromycin dihydrochloride (2 $\mu \mathrm{g} / \mathrm{ml}$; Amresco, Solon, OH, USA) was used to generate HeLa cell lines that stably express B7-H4 (pCMV-B7-H4) and its negative control (pCMV-myc).

Cell proliferation assay. Cell viability was assessed using Cell Counting Kit-8 (CCK-8) (Tongren, Shanghai, China). According to the product manual, $2 \times 10^{3}$ cells were seeded in each well of a 96-well plate, and incubated for $0,24,48$, 72 and $96 \mathrm{~h}$, and $10 \mu \mathrm{l}$ of CCK-8 reagent was added to each well at $4 \mathrm{~h}$ before assessing the optical density (OD) at $450 \mathrm{~nm}$ using a microplate reader (Infinite 2000; Tecan, Männedorf, Switzerland). 
Table I. Primers used in RT-PCR analysis and the small interfering RNA sequences.

\begin{tabular}{lll}
\hline Gene & & \multicolumn{1}{c}{ Primer sequence } \\
\hline B7-H4 & Forward & 5'-CCCAATCCGAAGTGTCAACT-3' \\
Si-B7-H4 & Reverse & 5'-TATCCTGGTGCCCGATAGAG-3' \\
& Forward & 5'-GUCACCUACAGCUGCUAAATT-3' \\
Si-control & Reverse & 5'-UUUAGCAGCUGUAGGUGACTT-3' \\
& Forward & 5'-UUCUCCGAACGUGUCACGUTT-3' \\
E7 & Reverse & 5'-ACGUGACACGUUCGGAGAATT-3' \\
& Forward & 5'-AGTGTGACTCTACGCTTCGG-3' \\
Rb & Reverse & 5'-TGTGCCCATTAACAGGTCTT-3' \\
& Forward & 5'-ATGCCCCAGAACCCTTGTATC-3' \\
MMP-2 & Reverse & 5'-GCCCATAGCCTTCCTTCTGAT-3' \\
& Forward & 5'-TACAGGATCATTGGCTACACACC-3' \\
MMP-9 & Reverse & 5'-GGTCACATCGCTCCAGACT-3' \\
VEGF & Forward & 5'-TGTACCGCTATGGTTACACCTCG-3' \\
& Reverse & 5'-GGCAGGGACAGTTGCTTCT-3' \\
GAPDH & Forward & 5'-TCTCTACCCCAGGTCAGACG-3' \\
& Reverse & 5'-AGCAATGTCCTGAAGCTCCC-3' \\
\hline
\end{tabular}

MMP, matrix metalloproteinase; VEGF, vascular endothelial growth factor.

Flow cytometry. Cells were washed twice using precooling PBS, and then digested in $75 \%$ alcohol at $4^{\circ} \mathrm{C}$ overnight. Propidium iodide (PI) was added into the cell suspensions and the cell cycle distribution was detected by FACSCalibur flow cytometer (both from BD Biosciences, Franklin Lakes, NJ, USA). As for cell apoptosis, we used two apoptosis kits, one was the Annexin V-PE/PI, the other was the Annexin V-FITC/7-AAD. The results were analyzed following the manufacturer's protocol using the flow cytometer as aforementioned.

Transwell migration and invasion assays. After the transfection of Si-B7-H4 or pCMV-B7-H4 as well as their negative controls, $6 \times 10^{4} \mathrm{SiHa}$ or $4 \times 10^{4} \mathrm{HeLa}$ cells were digested and suspended in $100 \mu \mathrm{l}$ serum-free MEM and DMEM, respectively, then the cells were seeded into the upper Transwell chamber (8.0- $\mu \mathrm{m}$ pore size; Costar, Cambridge, MA, USA), in the absence or presence of $100 \mu 1$ of Matrigel (1:8 dilution in serum-free medium; Corning, Corning, NY, USA). Medium with $20 \%$ FBS was added to the lower chamber as a chemoattractant. After $24 \mathrm{~h}$, the cells passing through the filter were stained with $0.1 \%$ crystal violet, and the images were captured by the Olympus IX51 inverted microscope. The number of migrated or invaded cells was counted in five random fields (magnification, x200) of each chamber under the microscope.

Statistical analysis. GraphPad Prism version 5.01 (GraphPad Software Inc., San Diego, CA, USA) was used for statistical analysis. In the present study, data are expressed as the means with standard deviations (SDs), and statistical comparisons were performed using a Student's t-test or a Chi-squared test. $\mathrm{P}<0.05$ was considered to indicate a statistically significant result.

\section{Results}

Expression of B7-H4 in human cervical cancer tissues and cell lines. To examine whether $\mathrm{B} 7-\mathrm{H} 4$ is expressed in cervical cancer, the relative protein expression levels of $\mathrm{B} 7-\mathrm{H} 4$ in 19 normal cervical and 20 cervical cancer tissues were assessed by western blotting (Fig. 1A). The protein level of B7-H4 in the cervical cancer samples was significantly higher than that in the normal tissues $(\mathrm{P}<0.001)$. We also detected the B7-H4 protein expression in $\mathrm{H} 8$ and three cervical cancer cell lines including CaSki, HeLa and SiHa (Fig. 1C). The protein expression level of B7-H4 was the lowest in the NUC cells and the highest in the SiHa cell line. To investigate the cellular location of B7-H4, immunofluorescence staining (Fig. 2A) and immunohistochemical staining (Fig. 2B) were performed and the results suggested that B7-H4 was mainly distributed in the cytoplasm of the cervical cancer cells. Collectively, these data suggest that B7-H4 was highly expressed in the cytoplasm of cervical cancer cells. However, it was not equally expressed in the different cervical cancer cell lines. Thus, knockdown of B7-H4 in the SiHa cell line and overexpression of it in the HeLa cell line was employed to study the functions of B7-H4.

Expression of sB7-H4 in CIN and cervical cancer patients. To investigate the expression of B7-H4 in blood, ELISA assay was performed to detect the sB7-H4 in 15 healthy volunteers, $20 \mathrm{CIN}$ and 20 cervical cancer patients. The results (Fig. 3A; Table II) revealed that the level of sB7-H4 in CIN patients was higher than that of the healthy volunteers (Fig. 3A, 2.654 \pm 1.533 vs. $1.000 \pm 0.557 ; \mathrm{P}=0.0004)$, and in cervical cancer patients, $\mathrm{sB} 7-\mathrm{H} 4$ was higher than that of the CIN patients (Fig. 3A, 5.042 \pm 4.336 vs. $2.654 \pm 1.533 ; \mathrm{P}=0.0257$ ) and healthy volunteers (Fig. $3 \mathrm{~A}$, 

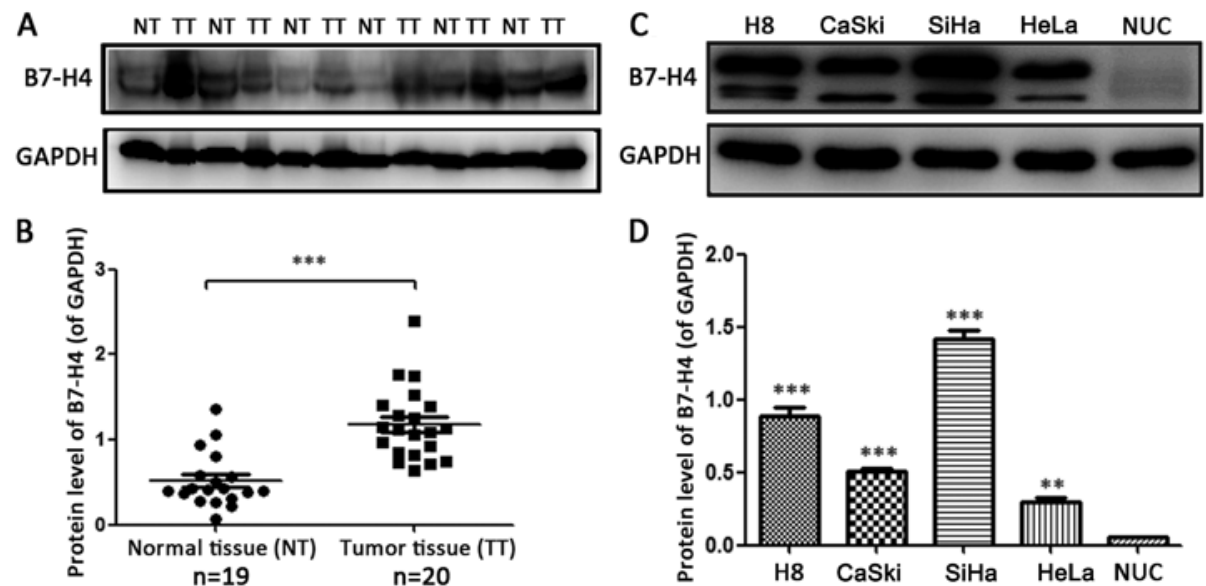

Figure 1. B7-H4 expression in normal and cervical cancer tissues and cervical cancer cell lines. (A) The protein expression of B7-H4 was determined by western blotting in 19 normal cervical tissues (NT) and 20 tumor tissues (TT). (B) Quantification of the protein expression levels as shown in A; ${ }^{* * * *} \mathrm{P}<0.001$. (C) The protein levels of B7-H4 were determined by western blotting in the H8, CaSki, HeLa, SiHa cell lines and normal uterine cervix (NUC) cells. (D) Quantification of the protein expression levels as shown in $C ;{ }^{* *} \mathrm{P}<0.01,{ }^{* * * *} \mathrm{P}<0.001$ compared with the NUC cells.
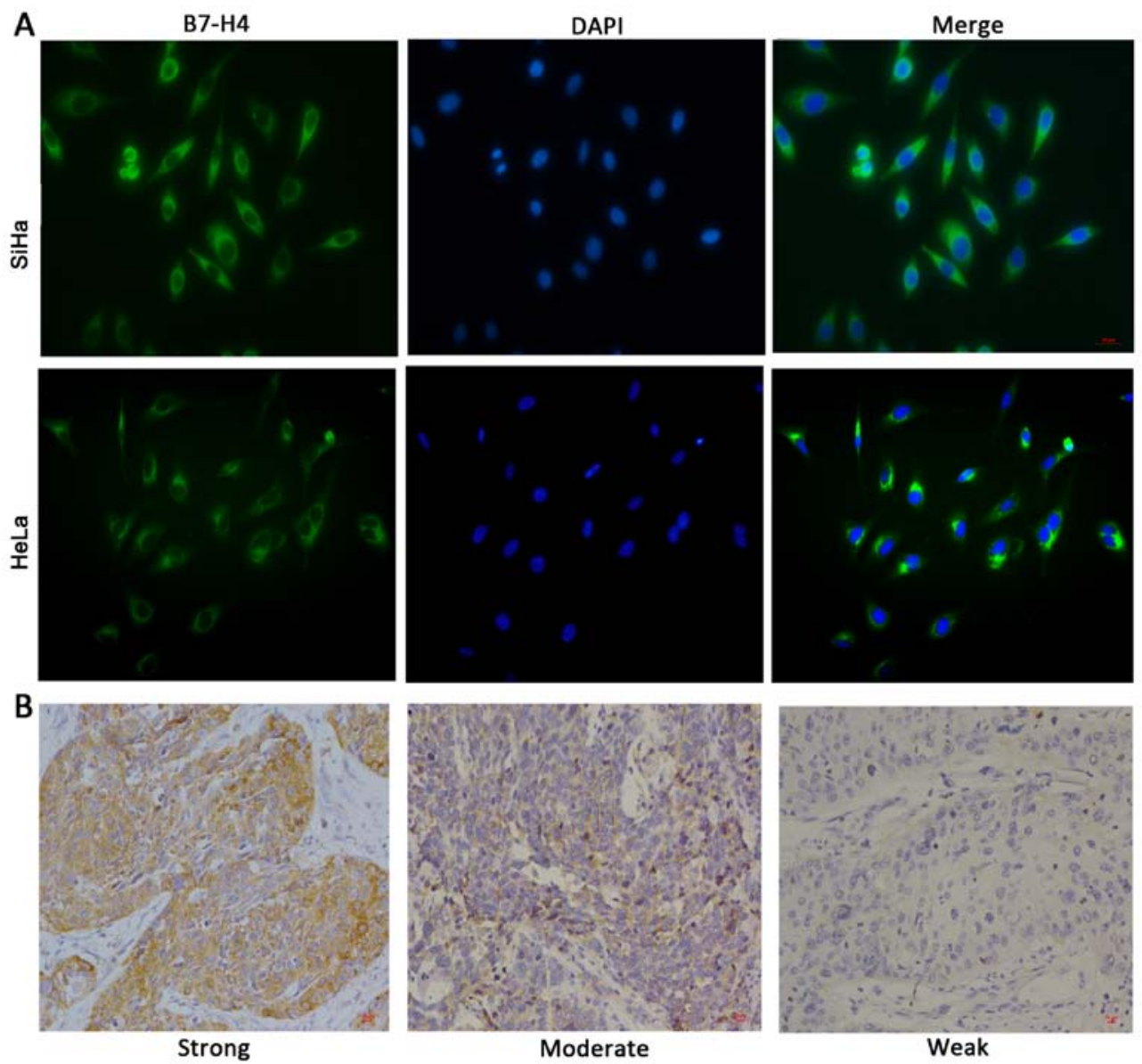

Figure 2. Subcellular distribution or location of B7-H4 in tumor cell lines SiHa and HeLa and in tumor tissue. (A) The SiHa and HeLa cells were stained with B7-H4 to label the cell cytoplasm and with DAPI to label the nucleus (magnification, x200). (B) Immunohistochemical staining of B7-H4 in tumor tissues exhibiting strong, moderate and weak expression (magnification, x200).

$5.042 \pm 4.336$ vs. $1.000 \pm 0.557 ; \mathrm{P}=0.0011)$. There was no difference in CIN I and CIN II-III $(\mathrm{P}=0.469)$ or in stage I and II cervical cancer patients $(\mathrm{P}=0.205)$, but the level of $\mathrm{sB} 7-\mathrm{H} 4$ in adenocarcinoma was higher than that in squamous carcinoma patients (Table II, $7.101 \pm 7.568$ vs. $4.527 \pm 3.307 ; \mathrm{P}=0.044$ ). The receiver operating characteristic curve (ROC) is presented in
Fig. 3B, and the area under the curve (AUC) was 0.955 , and by using the concentration of $\mathrm{sB} 7-\mathrm{H} 4 \geq 1.638$ as a critical value to predict CIN and cervical cancer, its sensitivity and specificity were 93.33 and $87.50 \%$, respectively. Collectively, these data demonstrated that $\mathrm{sB} 7-\mathrm{H} 4$ has the potential to become an early diagnostic indicator of cervical cancer. 
A

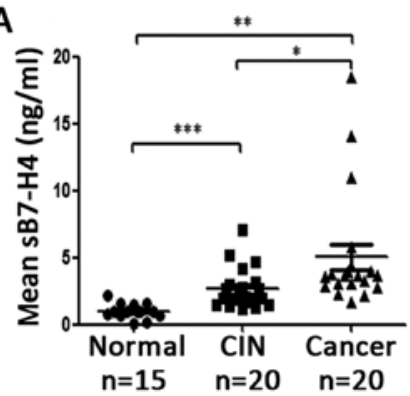

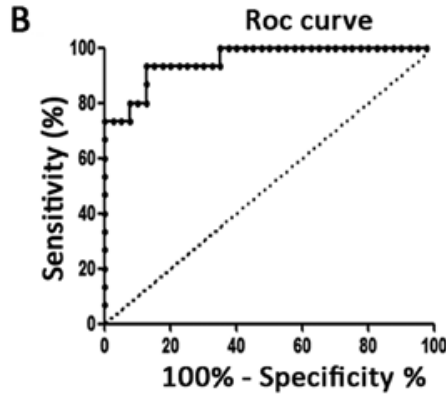

Figure 3. Expression level of B7-H4 in serum samples. (A) Distribution of serum B7-H4 in healthy women, cervical intraepithelial neoplasia (CIN) and cervical cancer patients; ${ }^{*} \mathrm{P}<0.05,{ }^{* *} \mathrm{P}<0.01,{ }^{* * *} \mathrm{P}<0.001$. (B) ROC curve for $\mathrm{B} 7-\mathrm{H} 4$ where the AUC was 0.955 .

Table II. Expression of sB7-H4 in CIN and cervical cancer patients and the relationship with their grades and histologies.

\begin{tabular}{lrccr}
\hline & \multicolumn{3}{c}{ Expression of sB7-H4 } & \multirow{2}{nnn}{ Subjects } \\
\cline { 3 - 4 } & No. & $\begin{array}{c}\text { Mean } \\
(\mathrm{ng} / \mathrm{ml})\end{array}$ & SD & P-value \\
\hline Normal-control & 15 & 1.000 & 0.557 & \\
CIN & 20 & 2.654 & 1.533 & \\
CIN I (LSIL) & 5 & 2.141 & 0.783 & 0.469 \\
CIN II/III (HSIL) & 15 & 2.782 & 1.663 & \\
Cervical cancer & 20 & 5.042 & 4.336 & \\
Stage I & 15 & 5.571 & 0.690 & 0.205 \\
Stage II & 5 & 3.452 & 4.916 & \\
Squamous carcinoma & 15 & 4.527 & 3.307 & $0.044^{\mathrm{a}}$ \\
Adenocarcinoma & 5 & 7.101 & 7.56 & \\
\hline
\end{tabular}

CIN, cervical intraepithelial neoplasia; LSIL, low-grade squamous intraepithelial lesion; HSIL, high-grade squamous intraepithelial

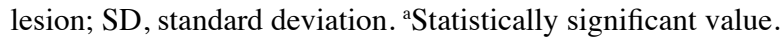

Relationship between B7-H4 expression in cervical cancer tissues and clinicopathological factors. Next, we performed immunohistochemical staining and the results are listed in Table III. We found that the expression level of B7-H4 in cervical cancer tissues was not correlated with age $(\mathrm{P}=0.241)$ histology $(\mathrm{P}=0.536)$, tumor differentiation $(\mathrm{P}=0.419)$, clinical stage $(\mathrm{P}=0.540)$, tumor size $(\mathrm{P}=0.183)$, lymph vascular space involvement (LVSI; $\mathrm{P}=1.000$ ), lymph node metastasis (LNM; $\mathrm{P}=0.620$ ) and deep stromal invasion (DSI; $\mathrm{P}=0.993$ ).

Effects of B7-H4 silencing and overexpression on E7/Rb $m R N A$. The HPV oncoprotein E7 forms a complex with tumor suppressor $\mathrm{Rb}$ and inhibits the activities of the proteins in cell cycle regulatory systems, which is essential for immortalization and transformation of human cervical squamous epithelial cells $(20,21)$. To investigate whether B7-H4 could affect $\mathrm{E} 7 / \mathrm{Rb}$ at the transcriptional level, we examined the effect of silencing and overexpression of B7-H4 on the mRNA level of E7/Rb. Compared with the control groups, the B7-H4 protein levels were significantly decreased in the SiHa cell line (Fig. 4A and B) and markedly increased in the HeLa cell line (Fig. 4C and D) by 2 -fold. In addition, the silencing of
Table III. Relationship between B7-H4 expression and clinicopathological factors.

Expression of B7-H4

\begin{tabular}{lrrrr} 
Clinicopathological & & & \\
factors & No. & Negative & Positive & P-value \\
\hline Age (years) & & & & 0.241 \\
$\quad \leq 45$ & 35 & 26 & 9 & \\
$>45$ & 25 & 15 & 10 & \\
Histology & & & & 0.536 \\
$\quad$ SCC & 53 & 35 & 18 & \\
$\quad$ Adenocarcinoma & 7 & 6 & 1 & \\
Differentiation & & & & 0.419 \\
Low & 27 & 17 & 10 & \\
$\quad$ Moderate/high & 33 & 24 & 9 & \\
Clinical stage & & & & 0.540 \\
I & 46 & 30 & 16 & \\
II & 14 & 11 & 3 & \\
Tumor size (cm) & & & & 0.183 \\
$<4$ & 42 & 26 & 16 & \\
$\geq 4$ & 18 & 15 & 3 & \\
LVSI & & & & 1.000 \\
$\quad$ Negative & 52 & 36 & 16 & \\
Positive & 8 & 5 & 3 & \\
LNM & & & & 0.620 \\
$\quad$ Negative & 50 & 33 & 17 & \\
Positive & 10 & 8 & 2 & \\
DSI & & & & 0.993 \\
$\geq 1 / 2$ & 22 & 15 & 7 & \\
$<1 / 2$ & 38 & 26 & 12 & \\
\hline
\end{tabular}

SCC, squamous cell carcinoma; LVSI, lymph vascular space involvement; LNM, lymph node metastasis; DSI, deep stromal invasion.

B7-H4 in the SiHa cell line resulted in the downregulation of E7 mRNA and the upregulation of Rb mRNA, and the overexpression of B7-H4 in the HeLa cell line led to the opposite effect (Fig. 4E and F). These findings revealed that B7-H4 may take part in the formation of cervical cancer by influencing the $\mathrm{E} 7 / \mathrm{Rb}$ pathway. 
A

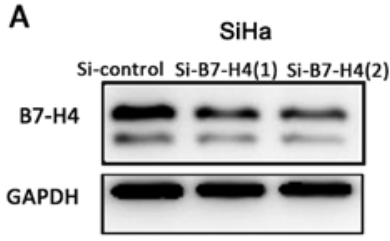

B

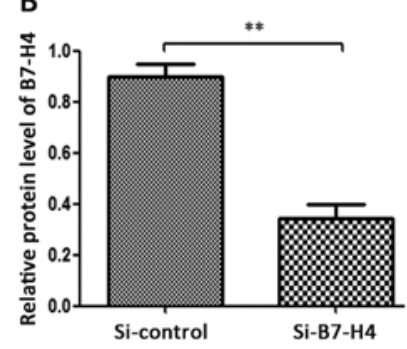

C

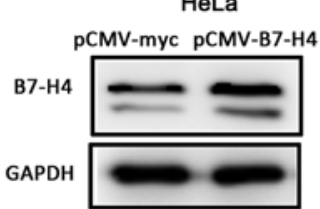

D

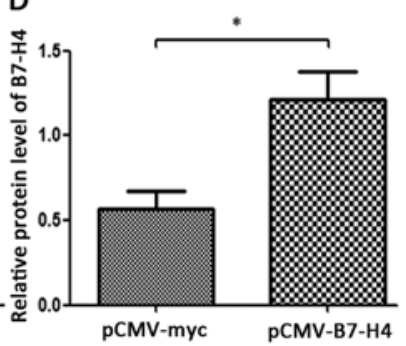

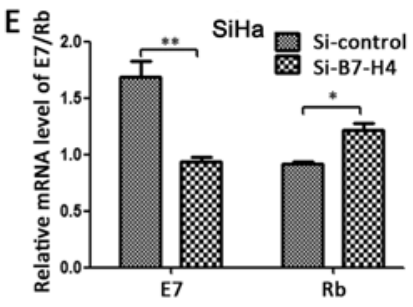

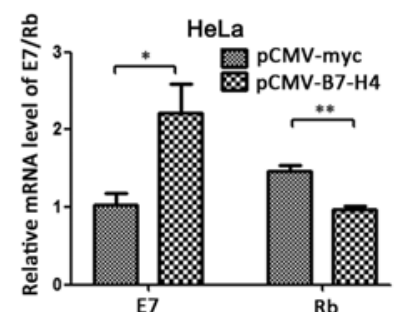

Figure 4. Effect of B7-H4 silencing and overexpression on E7/Rb mRNA. (A and C) The protein expression of B7-H4 in SiHa and HeLa cell lines after Si-B7-H4 or pCMV-B7-H4 transfection and their control groups was quantified by western blotting. (B and D) Quantification of the protein expression levels as shown in A and C. (E and F) RT-PCR was performed to identify the mRNA levels of E7 and Rb after the silencing or overexpression of B7-H4 in the SiHa and HeLa cell lines, respectively; ${ }^{*} \mathrm{P}<0.05,{ }^{* *} \mathrm{P}<0.01$ compared with the control groups.
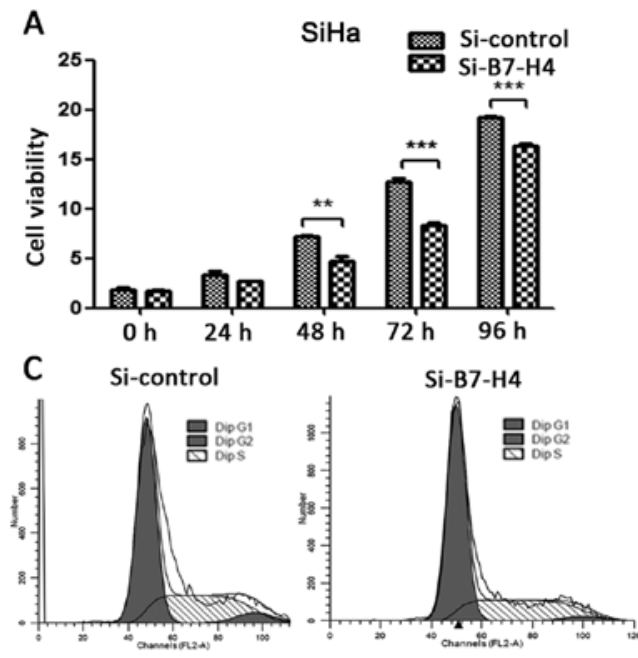

$\mathrm{E}$
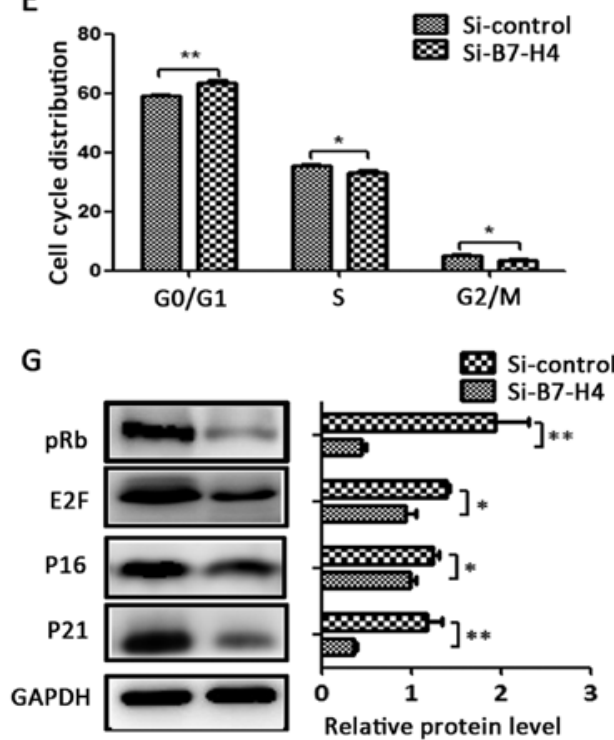

B HeLa

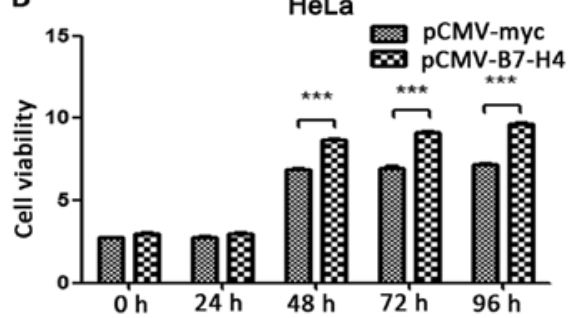

D pCMV-myc

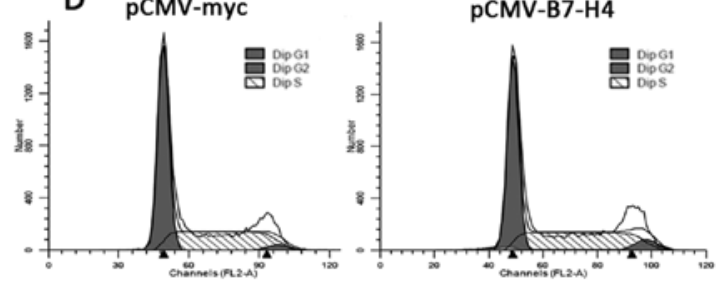

$\mathrm{F}$
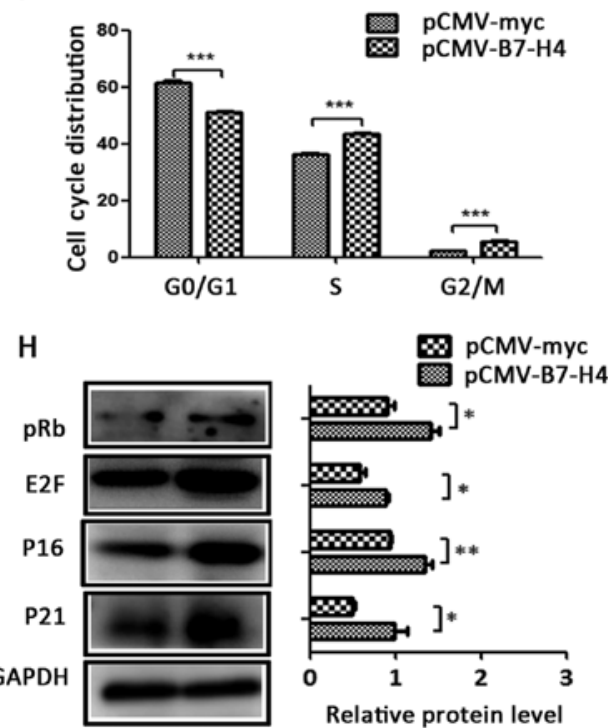

Figure 5. Effect of B7-H4 on cell viability and cell cycle distribution. (A and B) After siRNA or pCMV-B7-H4 transfection for 0, 24, 48, 72 and 96 h, the cell viability of the SiHa and HeLa cell lines was assessed using CCK-8 assay. (C and D) Cell cycle distributions of cells transfected with siRNA or pCMV-B7-H4 and their control groups were assessed by flow cytometry. (E and F) Cell cycle phase distribution was expressed as the percentage of total cells as shown in C and $\mathrm{D}$. ( $\mathrm{G}$ and $\mathrm{H}$ ) After siRNA or pCMV-B7-H4 transfection, the protein expression levels of pRb, E2F, P16 and P21 were determined and analyzed by western blotting; ${ }^{*} \mathrm{P}<0.05,{ }^{* *} \mathrm{P}<0.01,{ }^{* * *} \mathrm{P}<0.001$ compared with the control groups. 
A

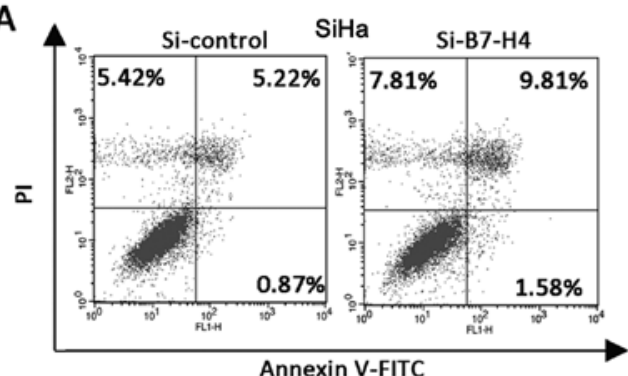

C

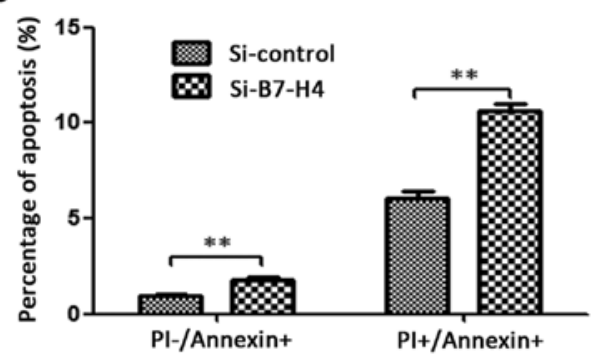

E

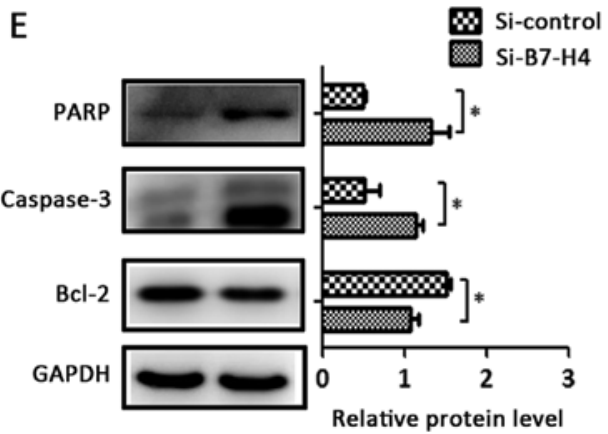

B

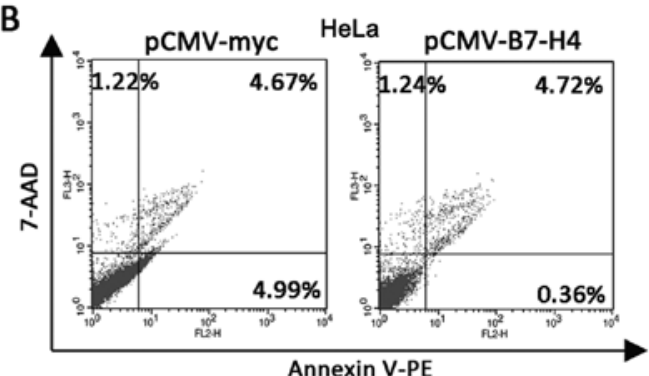

D

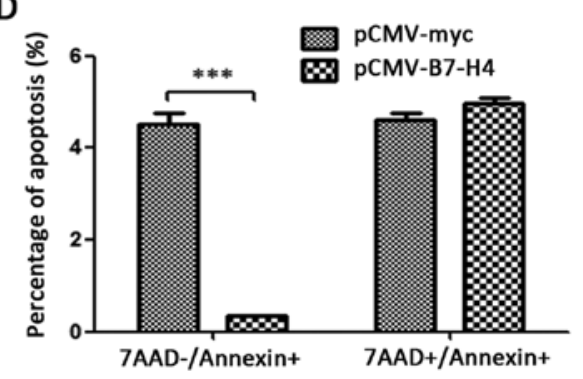

F

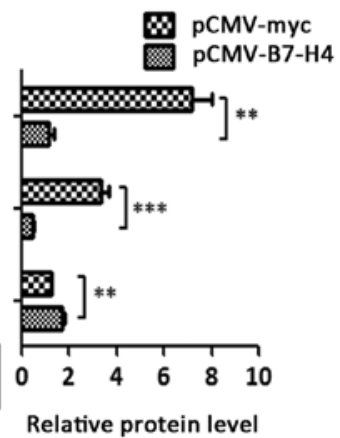

Figure 6. Effect of B7-H4 on cell apoptosis. (A) Knockdown of B7-H4 by siRNA induced the early and late apoptosis of the Si-B7-H4 SiHa cell line. (B) Overexpression of B7-H4 decreased the early apoptosis of the pCMV-B7-H4 HeLa cell line. (C and D) Quantification of the western blots of A and B. (E and F) After siRNA or pCMV-B7-H4 transfection, the protein expression levels of cleaved PARP, cleaved caspase-3 and Bcl-2 were analyzed by western blotting. ${ }^{*} \mathrm{P}<0.05,{ }^{* *} \mathrm{P}<0.01,{ }^{* * *} \mathrm{P}<0.001$ compared with the control groups.

B7-H4 increases cell viability and accelerates the cell cycle. To examine whether B7-H4 could affect cell viability and the cell cycle, we performed CCK-8 and flow cytometric assays (15). The cell viability of the $\mathrm{SiHa} \mathrm{Si}-\mathrm{B} 7-\mathrm{H} 4$ group was decreased at 48, 72 and $96 \mathrm{~h}$ (Fig. 5A), and the cell viability of the HeLa pCMV-B7-H4 group was increased at 48, 72 and $96 \mathrm{~h}$ (Fig. 5B), in comparison with their control groups. We also detected the cell cycle distribution using flow cytometry. Compared with the control groups, the $\mathrm{SiHa}$ cells treated with Si-B7-H4 were arrested in the G0/G1 phase (Fig. 5C) and the HeLa cells with overexpression of B7-H4 demonstrated accelerated $\mathrm{S}$ to $\mathrm{G} 2 / \mathrm{M}$ phase transition (Fig. 5D). We also found that the cell cycle regulatory proteins, including $\mathrm{pRB}$, E2F, P16 and P21, were influenced by B7-H4 expression. Specifically, the protein expression levels of pRB, E2F, P16 and P21 were altered with the expression change of B7-H4. These proteins were downregulated in the SiHa cells with silenced B7-H4 (Fig. 5G) and upregulated in the pCMV-B7-H4 group of the HeLa cell line (Fig. 5H) in comparison with their control groups.

B7-H4 inhibits cell apoptosis. To determine whether B7-H4 expression affects cervical cancer cell death, the knock- down of B7-H4 in the SiHa cell line led to an increase in early apoptosis ( $\left.\mathrm{PI}^{-} / \mathrm{Annexin}^{+}\right)$as well as late apoptosis $\left(\mathrm{PI}^{+} / \mathrm{Annexin}^{+}\right.$) (Fig. 6A and C), and the overexpression of B7-H4 in the HeLa cell line led to a decrease of early apoptosis (7AAD $/ \mathrm{Annexin}^{+}$) (Fig. 6B and D) when compared with the control groups. The western blotting results revealed that the apoptosis-related proteins, including cleaved PARP and cleaved caspase-3, were upregulated in the Si-B7-H4 group of the SiHa cell line (Fig. 6E) and downregulated in the pCMV-B7-H4 group of the HeLa cell line (Fig. 6F) in comparison with the control groups, and the anti-apoptosis protein Bcl-2 was in inverse proportion (Fig. 6E and F).

B7-H4 promotes migration and invasion. Compared with the control groups, the migrated and invasive number of cells decreased significantly in the SiHa Si-B7-H4 group and increased markedly in the HeLa pCMV-B7-H4 group (Fig. 7A and B). Matrix metalloproteinase (MMP)-2, MMP-9 and vascular endothelial growth factor (VEGF) have been reported to be associated with the migration and invasion of various cancer cell lines (22-24). To study whether B7-H4 affected MMP-2, MMP-9 and VEGF at the transcriptional level, we examined the effect of the silencing 

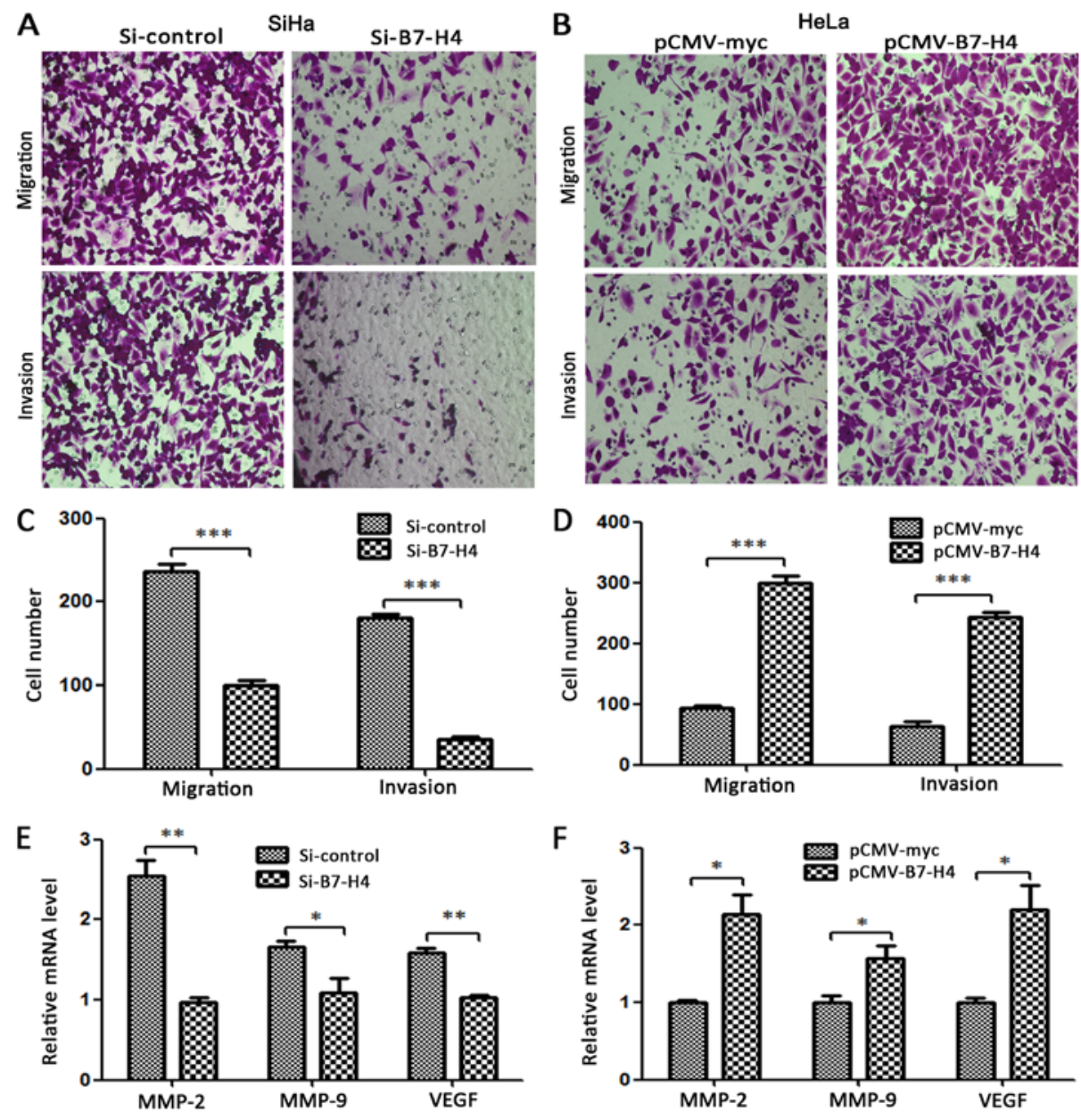

Figure 7. Effect of B7-H4 on the migration and invasion of the SiHa and HeLa cell lines. (A) Silencing B7-H4 in the SiHa cell line resulted in the decrease of migrated and invaded cells. (B) Overexpression of B7-H4 in the HeLa cell line increased the number of migrated and invaded cells. (C and D) Quantification of the images of A and B. (E and F) RT-PCR was performed to identify the mRNA levels of MMP-2, MMP-9 and VEGF after the silencing or overexpression of B7-H4 in the SiHa and HeLa cell lines, respectively; ${ }^{*} \mathrm{P}<0.05,{ }^{* *} \mathrm{P}<0.01,{ }^{* * *} \mathrm{P}<0.001$ compared with the control groups.

and overexpression of B7-H4 at the mRNA level of MMP-2, MMP-9 and VEGF, and found that these three molecules were regulated with the expression change of B7-H4 as determined by RT-PCR. Specifically, the silencing of B7-H4 in the SiHa cell line decreased the mRNA expression of these molecules (Fig. 7E) while the overexpression of B7-H4 in the HeLa cell line increased their mRNA expression (Fig. 7F).

\section{Discussion}

B7-H4 has been reported be involved in the occurrence and development of tumors through the regulation of the immune system $(7,8,25,26)$ and plays additional roles in the carcinogenic process directly $(15,16,27)$. In the present study, we provided evidence that $\mathrm{B} 7-\mathrm{H} 4$ expression is present in $\mathrm{CIN}$ and cervical cancer patients and we elucidated the potential underlying mechanism of their carcinogenic effect.

B7-H4 was mainly distributed in the cytoplasm of the cervical cancer cells according to our experimental results, and Zhang et al reported that B7-H4 was also a cytoplasmic-nuclear shuttling protein and could perform different functions in its different subcellar locations (27).

Through the detection of serum B7-H4 in healthy women, CIN and cervical cancer patients, we found that the AUC of
sB7-H4 was 0.955 , and the sensitivity and specificity reached 93.33 and $87.50 \%$ (Fig. 3), respectively. Compared with the most commonly used HPV testing $(28,29)$, the sensitivity was approximately the same, but the specificity was greatly improved. Thus, we tentatively conclude that serum B7-H4 could be used for the early detection and diagnosis of CIN and cervical cancer in the future; however, more direct human serum samples are essential to ascertain this conclusion. The results of immunohistochemistry (Table III) revealed that the expression of B7-H4 was not associated with clinicopathological characteristics such as age, histology, diffentation, clinical stage, tumor size, LVSI, LNM and DSI, which is consistent with previous studies $(12,30)$.

It is known that the $\mathrm{E} 7 / \mathrm{Rb}$ pathway plays a crucial role in the carcinogenic process caused by the persistent infection of high risk HPV (3). E7 is an HPV-derived oncogenic protein, and it combines with the tumor-suppressor molecule $\mathrm{Rb}$, leading to the release of transcripton activator E2F (3,31-33). Our data suggest that B7-H4 participates and influences this process: the silencing of $\mathrm{B} 7-\mathrm{H} 4$ in the $\mathrm{SiHa}$ cell line resulted in the increase of E7 mRNA and the decrease of Rb mRNA, along with an increase in the protein level of E2F, and the overexpression of B7-H4 in the HeLa cell line had the opposite effect (Figs. 4E and F, and 5G and $\mathrm{H}$ ). The follow-up proliferation assay results confirmed this hypothesis. However, the cell cycle 
regulation proteins phosphorylated $\mathrm{Rb}, \mathrm{P} 16$ and $\mathrm{P} 21$ were also altered with the regulation of $\mathrm{B} 7-\mathrm{H} 4$. The overexpression of $B 7-H 4$ protected cancer cells from apoptosis, and the silencing of B7-H4 enhanced intracellular caspase activity, leading to the acceleration of cervical cancer cell apoptosis. This can account for the alteration of apoptosis-related proteins including cleaved PARP and cleaved caspase- 3 as well as the anti-apoptosis protein Bcl-2. This finding was consistent with a study by Salceda et al (13). B7-H4 promoted the mRNA expression of MMP-2, MMP-9 and VEGF. According to previous studies, these molecules are related to the migration and invasion of tumor cells (22-24), suggesting that B7-H4 may be a potential therapeutic target for cervical cancer. We did not conduct a tumorigenicity assay in vitro due to the limitations of the experimental conditions, and the effect of B7-H4 on tumor formation in the LoVo colorectal cell line and in HEK293 cells has previously been confirmed by different groups $(15,27)$.

In conclusion, our findings suggest that the inhibitory molecule B7-H4 is involved in cervical cancer progression. Serum B7-H4 could be used as a valuable prognostic indicator for CIN and cervical cancer patients, and targeting B7-H4 may be a promising treatment of cervical cancer.

\section{Acknowledgements}

The present study was conducted at Qilu Hospital, Shandong University, and was supported by the Science and Technology Developing Planning of Shandong Province (2014GH218029), the National Natural Science Foundation of China (NSFC; 81572559), and the National Science and Technology Project of China (2015BAI13B05).

\section{References}

1. Torre LA, Bray F, Siegel RL, Ferlay J, Lortet-Tieulent J and Jemal A: Global cancer statistics, 2012. CA Cancer J Clin 65: 87-108, 2015.

2. Chen W, Zheng R, Baade PD, Zhang S, Zeng H, Bray F, Jemal A, Yu XQ and He J: Cancer statistics in China, 2015. CA Cancer J Clin 66: 115-132, 2016.

3. zur Hausen H: Papillomaviruses causing cancer: Evasion from host-cell control in early events in carcinogenesis. J Natl Cancer Inst 92: 690-698, 2000.

4. Shi Y, Li L, Hu Z, Li S, Wang S, Liu J, Wu C, He L, Zhou J, Li Z, et al: A genome-wide association study identifies two new cervical cancer susceptibility loci at $4 \mathrm{q} 12$ and $17 \mathrm{q} 12$. Nat Genet 45: 918-922, 2013.

5. Postow MA, Callahan MK and Wolchok JD: Immune checkpoint blockade in cancer therapy. J Clin Oncol 33: 1974-1982, 2015.

6. Zou W and Chen L: Inhibitory B7-family molecules in the tumour microenvironment. Nat Rev Immunol 8: 467-477, 2008.

7. Zang X, Loke P, Kim J, Murphy K, Waitz R and Allison JP: B7x: A widely expressed B7 family member that inhibits $\mathrm{T}$ cell activation. Proc Natl Acad Sci USA 100: 10388-10392, 2003.

8. Prasad DV, Richards S, Mai XM and Dong C: B7S1, a novel $\mathrm{B} 7$ family member that negatively regulates $\mathrm{T}$ cell activation. Immunity $18: 863-873,2003$

9. Sica GL, Choi IH, Zhu G, Tamada K, Wang SD, Tamura H, Chapoval AI, Flies DB, Bajorath J and Chen L: B7-H4, a molecule of the B7 family, negatively regulates $\mathrm{T}$ cell immunity. Immunity 18: 849-861, 2003.

10. Rahbar R, Lin A, Ghazarian M, Yau HL, Paramathas S, Lang PA, Schildknecht A, Elford AR, Garcia-Batres C, Martin B, et al: B7-H4 expression by nonhematopoietic cells in the tumor microenvironment promotes antitumor immunity. Cancer Immunol Res 3: 184-195, 2015.

11. Rahbar R and Ohashi PS: B7-H4 is a positive regulator of antitumor immunity. OncoImmunology 5: e1050575, 2015.
12. Sun Y, Wang Y, Zhao J, Gu M, Giscombe R, Lefvert AK and Wang X: B7-H3 and B7-H4 expression in non-small-cell lung cancer. Lung Cancer 53: 143-151, 2006.

13. Salceda S, Tang T, Kmet M, Munteanu A, Ghosh M, Macina R, Liu W, Pilkington G and Papkoff J: The immunomodulatory protein $\mathrm{B} 7-\mathrm{H} 4$ is overexpressed in breast and ovarian cancers and promotes epithelial cell transformation. Exp Cell Res 306: 128-141, 2005

14. Krambeck AE, Thompson RH, Dong H, Lohse CM, Park ES, Kuntz SM, Leibovich BC, Blute ML, Cheville JC and Kwon ED: B7-H4 expression in renal cell carcinoma and tumor vasculature: Associations with cancer progression and survival. Proc Natl Acad Sci USA 103: 10391-10396, 2006.

15. Peng HX, Wu WQ, Yang DM, Jing R, Li J, Zhou FL, Jin YF, Wang SY and Chu YM: Role of B7-H4 siRNA in proliferation, migration, and invasion of LOVO colorectal carcinoma cell line. BioMed Res Int 2015: 326981, 2015.

16. Cheng L, Jiang J, Gao R, Wei S, Nan F, Li S and Kong B: B7-H4 expression promotes tumorigenesis in ovarian cancer. Int $\mathrm{J}$ Gynecol Cancer 19: 1481-1486, 2009.

17. Simon I, Liu Y, Krall KL, Urban N, Wolfert RL, Kim NW and McIntosh MW: Evaluation of the novel serum markers B7-H4, Spondin 2, and DcR3 for diagnosis and early detection of ovarian cancer. Gynecol Oncol 106: 112-118, 2007.

18. Wang W, Xu C, Wang Y, Yu L and Zhang X: Prognostic values of B7-H4 in non-small cell lung cancer. Biomarkers: 1-16, 2016.

19. Pecorelli S, Zigliani L and Odicino F: Revised FIGO staging for carcinoma of the cervix. Int J Gynaecol Obstet 105: 107-108, 2009.

20. McMurray HR, Nguyen D, Westbrook TF and McAnce DJ: Biology of human papillomaviruses. Int J Exp Pathol 82: 15-33, 2001.

21. Kaur P, McDougall JK and Cone R: Immortalization of primary human epithelial cells by cloned cervical carcinoma DNA containing human papillomavirus type 16 E6/E7 open reading frames. J Gen Virol 70: 1261-1266, 1989.

22. Chen J, Lai L, Liu S, Zhou C, Wu C, Huang M and Lin Q: Targeting HIF-1 $\alpha$ and VEGF by lentivirus-mediated RNA interference reduces liver tumor cells migration and invasion under hypoxic conditions. Neoplasma 63: 934-940, 2016.

23. Lu X, Duan L, Xie H, Lu X, Lu D, Lu D, Jiang N and Chen Y: Evaluation of MMP-9 and MMP-2 and their suppressor TIMP-1 and TIMP-2 in adenocarcinoma of esophagogastric junction. Onco Targets Ther 9: 4343-4349, 2016.

24. Xie B, Zhang Z, Wang H, Chen Z, Wang Y, Liang H, Yang G, Yang $\mathrm{X}$ and Zhang $\mathrm{H}$ : Genetic polymorphisms in MMP 2, 3, 7 , and 9 genes and the susceptibility and clinical outcome of cervical cancer in a Chinese Han population. Tumour Biol 37: 4883-4888, 2016

25. Fauci JM, Straughn JM Jr, Ferrone S and Buchsbaum DJ: A review of $\mathrm{B} 7-\mathrm{H} 3$ and $\mathrm{B} 7-\mathrm{H} 4$ immune molecules and their role in ovarian cancer. Gynecol Oncol 127: 420-425, 2012.

26. Wang X, Wang T, Xu M, Xiao L, Luo Y, Huang W, Zhang Y and Geng W: B7-H4 overexpression impairs the immune response of $\mathrm{T}$ cells in human cervical carcinomas. Hum Immunol 75: 1203-1209, 2014

27. Zhang L, Wu H, Lu D, Li G, Sun C, Song H, Li J, Zhai T, Huang L, Hou C, et al: The costimulatory molecule $\mathrm{B} 7-\mathrm{H} 4$ promote tumor progression and cell proliferation through translocating into nucleus. Oncogene 32: 5347-5358, 2013.

28. Xu H, Lin A, Shao X, Shi W, Zhang Y and Yan W: Diagnostic accuracy of high-risk HPV genotyping in women with high-grade cervical lesions: Evidence for improving the cervical cancer screening strategy in China. Oncotarget 7: 83775-83783, 2016.

29. Park IU, Wojtal N, Silverberg MJ, Bauer HM, Hurley LB and Manos MM: Cytology and human papillomavirus co-test results preceding incident high-grade cervical intraepithelial neoplasia. PLoS One 10: e0118938, 2015.

30. Wang L, Cao NN, Wang S, Man HW, Li PF and Shan BE: Roles of coinhibitory molecules B7-H3 and B7-H4 in esophageal squamous cell carcinoma. Tumour Biol 37: 2961-2971, 2016.

31. Balsitis S, Dick F, Dyson N and Lambert PF: Critical roles for non-pRb targets of human papillomavirus type $16 \mathrm{E} 7$ in cervical carcinogenesis. Cancer Res 66: 9393-9400, 2006.

32. van der Watt PJ, Ngarande E and Leaner VD: Overexpression of $\mathrm{Kpn} \beta 1$ and Kpn $\alpha 2$ importin proteins in cancer derives from deregulated E2F activity. PLoS One 6: e27723, 2011.

33. Crosbie EJ, Einstein MH, Franceschi S and Kitchener HC: Human papillomavirus and cervical cancer. Lancet 382: 889-899, 2013. 